\title{
Caracterización clínica epidemiológica del cáncer de mama en mujeres mayores de 20 años en El Salvador
}

\author{
DOI 10.5377/alerta.v4i3.10952 \\ Julio Armando Orellana Beltrán ${ }^{1^{*}}$, Oscar Mauricio Valladares Martínez ${ }^{2}$ \\ 1. Facultad de Medicina, Universidad de El Salvador \\ 2. Aseguramiento de la Calidad, Instituto Salvadoreño del Seguro Social \\ ${ }^{*}$ Correspondencia \\ $\square$ dr.julioorellana@gmail.com \\ 1. (D) $0000-0003-1338-6267$
}

G

ACCESO ABIERTO

Clinical epidemiological characterization of breast cancer in women older than 20 years in $\mathrm{EI}$ Salvador

\section{Citación recomendada:}

Orellana Beltrán JA, Valladares

Martínez OM. Caracterización

clínica epidemiológica del

cáncer de mama en mujeres

mayores de 20 años en El

Salvador. Alerta. 2021;4(3):126-

134. DOI: $10.5377 /$ alerta.

v4i3.10952

Recibido:

1 de marzo de 2021

\section{Aceptado:}

6 de julio de 2021

\section{Publicado:}

26 de julio de 2021

\section{Contribución de autoría:} JAOB $^{1}$ : La idea del estudio, diseño metodológico,

implementación y conducción análisis de los resultados y escritura del artículo. OMVM²: Implementación y conducción, análisis de los resultados.

\section{Conflicto de intereses:}

Los autores declaran no tener conflictos de intereses

\begin{abstract}
Resumen
Introducción. El cáncer de mama para el año 2020 fue el número uno a nivel mundial, con una tendencia al alza constante. Puesto que afecta tanto a países desarrollados como en vía desarrollo, las consecuencias derivadas demandan al sistema de salud una respuesta oportuna. Objetivo. Caracterizar clínica y epidemiológicamente el cáncer de mama en las pacientes mayores de 20 años atendidas en el Instituto del Cáncer de El Salvador. Metodología. La investigación fue de tipo transversal descriptivo. Se recolectó información de 344 expedientes de pacientes con cáncer de mama de enero de 2017 a diciembre de 2018 con diagnóstico por biopsia de muestra patológica, mayores de 20 años. Luego se aplicó un análisis estadístico descriptivo. Resultados. Se identificó un mayor porcentaje, 59,9\%, de pacientes de zona urbana; el 89,2\% fue ama de casa, la edad promedio de diagnóstico de cáncer fue 54 años y el promedio de índice de masa corporal fue de 29,1. El signo clínico inicial fue tumoración, el 85,8\%, con un tamaño promedio de 4,8 cm. El diagnóstico más frecuente, equivalente al 83,4\%, fue carcinoma ductal invasivo y más del $50 \%$ fue $\geq$ N1. Se observó una mayor positividad de receptores hormonales. El $84,1 \%$ de las pacientes recibieron tratamiento con radioterapia. Conclusiones. Las mujeres con cáncer de mama tienen las siguientes características: adulta de la sexta década de la vida, con sobrepeso, ama de casa, con signo inicial de tumoración, en estadio local avanzado, con diagnóstico de carcinoma ductal invasivo.
\end{abstract}

Palabras clave

Neoplasias de la mama, epidemiología, factores de riesgo, clínica

\section{Abstract}

Introduction. By the year 2020, breast cancer was ranked number one in the world, with a constant upward trend, it affects developed and developing countries, the resulting consequences demand a timely response from the health system. Objective. To characterize clinically and epidemiologically breast cancer in patients older than 20 years treated at the Cancer Institute of El Salvador. Methodology. A cross sectional descriptive study was carried out, Information was collected from 344 records of female patients older than 20, with breast cancer from January 2017 to December 2018, diagnosed by biopsy of a pathological sample. A descriptive statistical analysis was applied. Results. A higher percentage, 59,9\%, of patients were identified in urban areas; $89,2 \%$ were housewives, the average age of cancer diagnosis was 54 years, the average Body Mass Index was 29,1. The initial clinical sign was a tumor, $85,8 \%$, with an average size of $4,8 \mathrm{~cm}$. The most frequent diagnosis, $83,4 \%$, was invasive ductal carcinoma, and more than $50 \%$ was $\geq \mathrm{N} 1$. Higher hormone receptor positivity was observed. $84,1 \%$ of the patients received radiotherapy treatment. Conclusion. Women with breast cancer have the following characteristics: an adult in the sixth decade of life, overweight, a housewife, with an initial sign of a tumor, in an advanced local stage with a diagnosis of invasive ductal carcinoma.

Keywords

Breast neoplasms, epidemiology, risk factor, clinical

\section{Introducción}

El cáncer de mama es un problema a nivel mundial que afecta tanto a países desarrollados y como en desarrollo, de los cuales estos últimos son los que presentan la mortalidad más alta. Este tipo de cán- cer fue el más frecuente para el año 2020 en todo el mundo. Según reportes de The Global Cancer Observatory (GCO), para el año 2020, alrededor del mundo ocurrieron 2261419 casos de cáncer de mama, es decir, el 11,7\% del total de cánceres, y 684996 muertes'. 
La tendencia de esta enfermedad es al alza constante y las consecuencias derivadas demandan al sistema de salud una respuesta oportuna. Estudios económicos estiman que el exceso de costo medio en atención para cáncer de mama fue de 36948 dólares australianos (AUD) por cada caso en el primer año posdiagnóstico ${ }^{2}$. El costo promedio anual después de la fase de tratamiento inicial por cada caso fue de AUD 4023, y durante la fase terminal, de AUD $38619^{2}$.

La Agencia Internacional para la Investigación del Cancer registró un total de 210 100 casos de cáncer de mama en la región de Latinoamérica y el Caribe, en el año 2020, esto representa el 9,3\% del total de cáncer de mama en el mundo, mientras que la mortalidad representa un $8,5 \%$ del total de muertes por cáncer de mama en el mundo3. En El Salvador, para el año 2018, el cáncer de mama representó el 14,4\% del total de cánceres, con una tasa de incidencia de cáncer de mama en mujeres fue de 39,9 casos por 100000 habitantes, en comparación con el año 2020, hubo un aumento de los casos diagnosticados de cáncer de mama, por lo que paso a representar el $16,4 \%$ del total de cánceres, colocándose el cáncer en el número uno en el país. En ese año, la tasa de incidencia de cáncer de mama en mujeres fue de 40,5 casos por 100000 habitantes $^{3}$.

El número de casos nuevos de cáncer de mama registrados en El Salvador en el periodo de 2013 a 2018 fue de 3970, con una tasa de mortalidad de 10,9 casos por 100000 habitantes y un número total de defunciones de 427, mientras que en el periodo de 2015 a 2020 hubo una disminución en el número de casos nuevos, de 2767 casos con una tasa de mortalidad de 8,1 casos por 100000 habitantes y un total de 331 defunciones ${ }^{3}$.

Durante los años 2017 y 2018, el ICES recibió un total de 404 mujeres con diagnóstico de cáncer de mama, de estas el 79,7\% fueron referidas en al 2017.

Este comportamiento de la enfermedad ha generado la necesidad de conocer sobre la caracterización clínica y epidemiológica del cáncer de mama en El Salvador.

\section{Metodología}

Se llevó a cabo un estudio transversal descriptivo en 344 pacientes con cáncer de mama, identificadas a partir de la revisión de un total de 404 expedientes clínicos clasificados por el Departamento de estadísticas del Instituto del Cáncer de El Salvador (ICES), como referencias por cáncer de mama correspondientes al periodo que comprende del 1 de enero de 2017 al 31 de diciembre de 2018.
Los criterios de inclusión fueron: pacientes con edad mayor de 20 años, expediente con diagnóstico histopatológico de cáncer de mama, el periodo de referencia al ICES entre 1 de enero de 2017 al 31 de diciembre de 2018. Los criterios de exclusión fueron: expediente incompleto que comprometa más del $60 \%$ de las variables, expediente deteriorado o ilegible, nacionalidad extranjera, tumor benigno de mama, cáncer no primario de mama.

Las variables incluidas en este estudio fueron: área de procedencia, zona de residencia, ocupación, edad, peso, talla, IMC, paridad, número de hijos, cuadro clínico inicial, tamaño de tumor, estadio de la enfermedad ${ }^{4}$, tipo histológico del cáncer, marcador inmunohistoquímico del cáncer, puntuación de Karnofsky, cumplimiento de tratamiento, tipo de tratamiento y tipo de radioterapia.

El tamaño de la muestra correspondió al total de la población que cumplió los criterios de inclusión. Asimismo, los expedientes fueron agregados de forma consecutiva. De los 404 expedientes clínicos clasificados como referencias por cáncer de mama primario como consulta de primera vez, se excluyeron 60 expedientes por razones como: no reporte de biopsia, otro tipo de cáncer, tumor benigno, menores de 20 años o expediente incompleto.

Posterior a la autorización de la dirección del ICES y al apoyo del departamento de estadística y archivo clínico, se efectuó la revisión y recolección de información de los expedientes clínicos; se revisaron en promedio 40 expedientes diarios, hasta completar el número de 404, obteniendo una muestra final de 344 que entraron al estudio.

La recolección de datos fue efectuada en su mayor parte por uno de los investigadores mediante un formulario de recolección de datos (FRD) digital usando la aplicación KoBoToolbox, instalada en dispositivos electrónicos móviles. Cada formulario fue codificado, protegiendo la integridad y confidencialidad de la información contenida en los expedientes de las pacientes.

\section{Métodos estadísticos}

Se descargó cada documento en un formato de archivo tipo SQL, CSV o.XIsX, se procesaron los datos en Microsoft Excel ${ }^{\circledR}$ versión 2016 y software Statistical Package for the Social Sciences (SPSS) versión 23. En la base de datos final se revisaron inconsistencias y luego se aplicó un análisis estadístico descriptivo, en el cual las variables cuantitativas se presentaron en medidas de tendencia central y dispersión, y las variables categóricas se presentaron en frecuencias y porcentaje. 


\section{Aspectos éticos}

La investigación cumplió con los principios éticos establecidos en la declaración de Helsinki. Se elaboró una acta de confidencialidad y protección de información requerida por las autoridades del ICES, para garantizar el adecuado uso de los datos.

\section{Resultados}

\section{Características epidemiológicas}

La distribución geográfica del total de pacientes incluidas en el estudio muestra un mayor porcentaje de pacientes con cáncer de mama procedentes de San Salvador $(25,3 \%)$, seguido por los departamentos de La Libertad (12,2 \%), Santa Ana (11 \%), San Miguel $(10,5 \%)$ y Cabañas $(1,5 \%)$. En cuanto a la zona de residencia, el 59,9\% de casos fueron de la zona urbana.

La ocupación de ama de casa representó el 89,2\%, el mayor porcentaje. El 2,9\% indicó ser comerciante; el 1,7\%, costurera; el 1,2\%, estudiante, y el 0,6\%, empleada y enfermera, el mismo porcentaje para cada una. Las ocupaciones y profesiones de abogada, arquitecta, cosmetóloga, decoradora, propietaria de negocio, odontóloga, panificadora, secretaria y otra, representaron el 0,3\% para cada de estas.

La edad promedio de diagnóstico de cáncer fue 54 años (DE 13), con un mínimo de 22 años y un máximo de 87 años. El diagnóstico de cáncer de mama se realizó con mayor frecuencia en pacientes con edad igual o mayor a 50 años. (Tabla 1)

Tabla 1. Edad de diagnóstico del cáncer de mama

\begin{tabular}{lrr}
\hline Edad & $\begin{array}{c}\text { Frecuencia } \\
(\mathrm{n})\end{array}$ & $\begin{array}{c}\text { Porcentaje } \\
(\%)\end{array}$ \\
\hline <35 años & 22 & 6,4 \\
35-39 años & 23 & 6,7 \\
40-44 años & 28 & 8,1 \\
45-49 años & 56 & 16,3 \\
> a 50 años & 215 & 62,5 \\
\hline
\end{tabular}

En cuanto al estado nutricional de las pacientes, el sobrepeso se presenta en el 32\% de las pacientes; el 20,3\% fue clasificado en obesidad grado l; el 16,6\% correspondió a un peso normal; y obesidad grado III, en el $3,2 \%$. El índice de masa corporal promedio fue de $81,4 \%$ de registros con información, el promedio fue de 29,1 (DE 5,2), con un mínimo de 16,6 y un máximo de 45,9.

El $27,3 \%$ de las pacientes parieron tres o más hijos, el 12,5\%, dos; el 27\%, uno, y el 5,2\% no tienen hijos. Sin embargo, el 27,9\% no te- nía datos en los expedientes clínicos que evidenciaran si las pacientes habían parido.

\section{Características clínicas}

En el cuadro clínico inicial se observó que con mayor frecuencia se reportó como signo inicial tumoración/masa (85,4\%), seguido de los nódulos (6,3\%) y el dolor (5,3\%). El resto de signos y síntomas se presentó en menos del $1 \%$.

Tabla 2. Cuadro clínico inicial de las pacientes con cáncer de mama

\begin{tabular}{lrr}
\hline Signos/Síntomas & $\begin{array}{r}\text { Frecuencia } \\
\text { (n) }\end{array}$ & $\begin{array}{r}\text { Porcentaje } \\
\text { (\%) }\end{array}$ \\
\hline Tumoración/masa & 339 & 85,4 \\
Nódulo & 25 & 6,3 \\
Dolor & 21 & 5.3 \\
Inversión de pezón & 5 & 1.3 \\
Telorragia/ & 3 & 0.8 \\
descargas de pezón & & \\
Retracción de piel & 2 & 0.5 \\
Absceso & 1 & 0.3 \\
Telangiectasia/ & & \\
neovascularización & \multicolumn{2}{c}{0.3} \\
\hline
\end{tabular}

En la Tabla 2 se observa la frecuencia de los signos y síntomas identificados en las pacientes, de forma individual. El 85,4\% de las pacientes refde mayor a menor: tumoración/masa, nódulo, dolor, inversión de pezón. El tamaño promedio del tumor o masa de mama fue de $4,8 \mathrm{~cm}$ (DE 2,8), con un mínimo de $0,5 \mathrm{~cm}$ y un máximo de $16,1 \mathrm{~cm}$. El $41 \%$ se presentó con tumoración/masa entre $>2 \mathrm{~cm}-\leq 5 \mathrm{~cm}$; el $38,7 \%$ con un tamaño $>5 \mathrm{~cm}$ y el $16,9 \%$ con $\leq 2 \mathrm{~cm}$.

\section{Diagnóstico y manejo}

El tipo histológico de cáncer diagnosticado más frecuente $83,4 \%$ fue el carcinoma ductal invasivo (NOS), también conocido como carcinoma invasivo de ningún tipo especial (NST), mientras que se observó un 4,9\% de carcinoma lobular invasivo (ILC); estos datos indican que el carcinoma NST fue 17 veces mayor respecto al ILC. En cuanto al carcinoma ductal in situ, fue tres veces mayor respecto al carcinoma lobulillar in situ. El carcinoma mucinoso y el carcinoma papilar invasivo fueron menos frecuentes (Figura 1).

Según la estadificación por TNM registrado, el 29,1\% fue clasificado en T4, el 25,9\% en T3, el 20,9\% en T2 y el 6,1\% en T1. El estado ganglionar $\mathrm{N} 2$ fue del $31,7 \%$ y el $\mathrm{N} 1$ de 31,4\%; el $13,1 \%$ en NO y el 4,1\% en N3. La metástasis(M) reportada fue menor al $2 \%$. 
En relación a la expresión de marcadores inmunohistoquímicos de cáncer, de los registros que reportaron las pruebas se observó una mayor positividad de receptores hormonales (Figura 2). El 64,4\% fue receptor de estrógeno (ER) positivo; asimismo, el 35,5\% fue ER negativo. Para el receptor progesterona (PR) positivo y negativo se tuvo el $66,6 \%$ y el $33,3 \%$, respectivamente. La expresión del receptor 2 del factor de crecimiento epidérmico humano (HER2) ${ }^{5}$ positivo fue del $26,2 \%$ y el negativo, del $73,7 \%$. Al $47 \%$ de las pacientes no se les realizó esta prueba.

En cuanto a la evaluación funcional medida por la puntuación de Karnofsky, el mayor porcentaje, $67,4 \%$, se obtuvo con una puntuación de 100; el 15,1\%, entre 80-90; el 2\%, entre 60-70. Por otra parte, en el 15,4\% de los expedientes clínicos no se encontró registros.

Las pacientes que cumplieron el tratamiento indicado según los registros del expediente alcanzan el $85,8 \%$, mientras que el $14,2 \%$ no cumplió el tratamiento. De las pacientes que cumplieron el tratamiento, la mayor frecuencia fue encontrada con tratamiento de radioterapia, $84,1 \%$, seguido por quimioterapia y quirúrgico, con el $12,2 \%$ y el $10,5 \%$, respectivamente.

La mayor frecuencia de la radioterapia es acorde a los servicios que ofrece dicha institución especializada en radioterapia para cáncer. En cuanto a la terapia administrada de forma combinada o sola, el 72,9\% recibió radioterapia como único tratamiento. Por otra parte, se observó combinación de tratamiento quirúrgico con radioterapia en el $5,4 \%$ y quimioterapia con radioterapia en el $4,7 \%$ de los casos. Del tipo de radioterapia, la teleterapia fue la de mayor administración, comparada con la braquiterapia (Figura 3).

\section{Discusión}

En este estudio se investigó la caracterización clínica y epidemiológica de las pacientes con cáncer de mama. Las características con mayor frecuencia que se encontraron fueron pacientes en la sexta década de vida, con sobrepeso, procedentes del área urbana. El tamaño promedio de tumor fue de 4 cm, con invasión a ganglios, diagnóstico de NOS y en tratamiento con radioterapia. Los resultados mostraron que la caracterización del cáncer difiere con lo reportado en algunos estudios internacionales ${ }^{6-15}$, pero para su interpretación también debe tomarse en cuenta la particularidad del servicio que ofrece la institución.
En cuanto a las características epidemiológicas, la mayor parte de pacientes provenían de zonas urbanas, similar a lo reportado por Sofi N (2019) ; esto coincidió con un mayor porcentaje de casos del área de San Salvador, una de las ciudades con mayor urbanización. Es posible que este hecho esté ligado a la dificultad de acceso desde el interior del país, así como a barreras económicas para recibir la atención especializada en esta institución. Otra alternativa podría ser por la adopción de estilos de vida propicios de un ambiente urbano, como la inactividad física, la obesidad, el alcoholismo, el tabaquismo o factores reproductivos'.

A nivel mundial se reportan más casos de cáncer de mama a partir los 40 años $^{16}$. En un estudio en la India, la edad promedio de diagnóstico fue $47 \pm 10$ años, en mayor porcentaje en el grupo de los 35-65 años ${ }^{6}$. En la presente investigación fue de 54 años \pm 13 , con mayor frecuencia en el grupo de más de 50 años, por lo que difirió en cerca de una década con el resto del mundo y en particular con Asia. En estudios similares efectuados en Colombia y México, el promedio de edad de los casos nuevos se encontró en los 54 años, siendo análogo a lo encontrado en la presente investigación. Parece que los países comparten condiciones similares de la región de Latinoamerica ${ }^{7,17}$.

En estudios realizados en el Instituto Nacional de Perinatología de México, la ocupación en las pacientes con cáncer de mama se distribuye, de mayor a menor, en ama de casa, empleada, comerciante y estudian$\mathrm{te}^{8}$, coincidiendo con el mayor porcentaje reportado para la ocupación ama de casa. Pocas pacientes tenían la ocupación comerciante. Respecto a la ocupación ama de casa, pudiera estar relacionada con la característica de la edad, mayor de 50 años; por tanto, a esa edad y al encontrarse padeciendo esta enfermedad, tienen más restricciones para desempeñar un trabajo y mayor necesidad de disposición de tiempo para recibir el tratamiento de su enfermedad ${ }^{18}$.

Las poblaciones latinoamericanas se caracterizan por prevalencias de sobrepeso $u$ obesidad mayores al 70\% ${ }^{19,20}$. En la investigación realizada, el mayor porcentaje de pacientes correspondió a mujeres posmenopáusicas con sobrepeso u obesidad, similar a lo reportado en la región. En otros estudios, la categoría de IMC normal tiene la mayor frecuencia ${ }^{6}$. Este resultado puede sugerir que nuestra población tuvo un riesgo de cáncer de 1,5 a 2 veces más alto, caracterizándose por tener un IMC elevado y, por ende, más grasa productora de hormonas que aumentan en el riesgo de cancer ${ }^{9,21}$. 
Carcinoma invasivo de ningún tipo especial (NST)*

Carcinoma lobular invasivo (ILC)

Carcinoma mucinoso

Carcinoma ductal in situ (CDIS)

Tumor phllodes de mama, maligno

Carcinoma con diferenciación apocrina

Carcinoma papilar invasivo

Carcinoma lobular in situ (CLIS)

Otros tipos de cáncer de mama

*Carcinoma invasivo de ningún tipo especial

(NST) o ductal invasivo (NOS)

Figura 1. Tipo histológico de cáncer diagnosticado por patólogo.

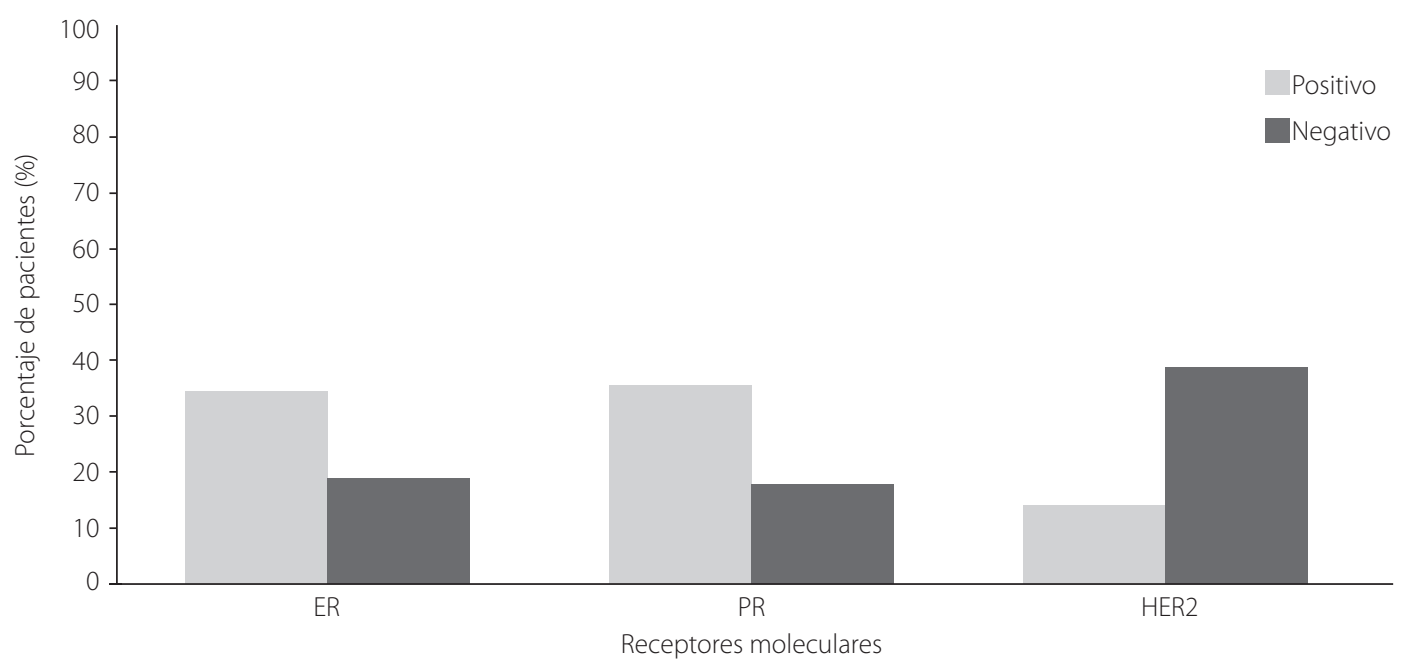

Figura 2. Expresión de marcadores inmunohistoquímicos en las pacientes con cáncer de mama. ER: receptor de estrógenos ( $n=183)$, PR: receptor de progesterona $(n=183)$, HER2: receptor 2 del factor de crecimiento epidérmico humano $(n=179)$.

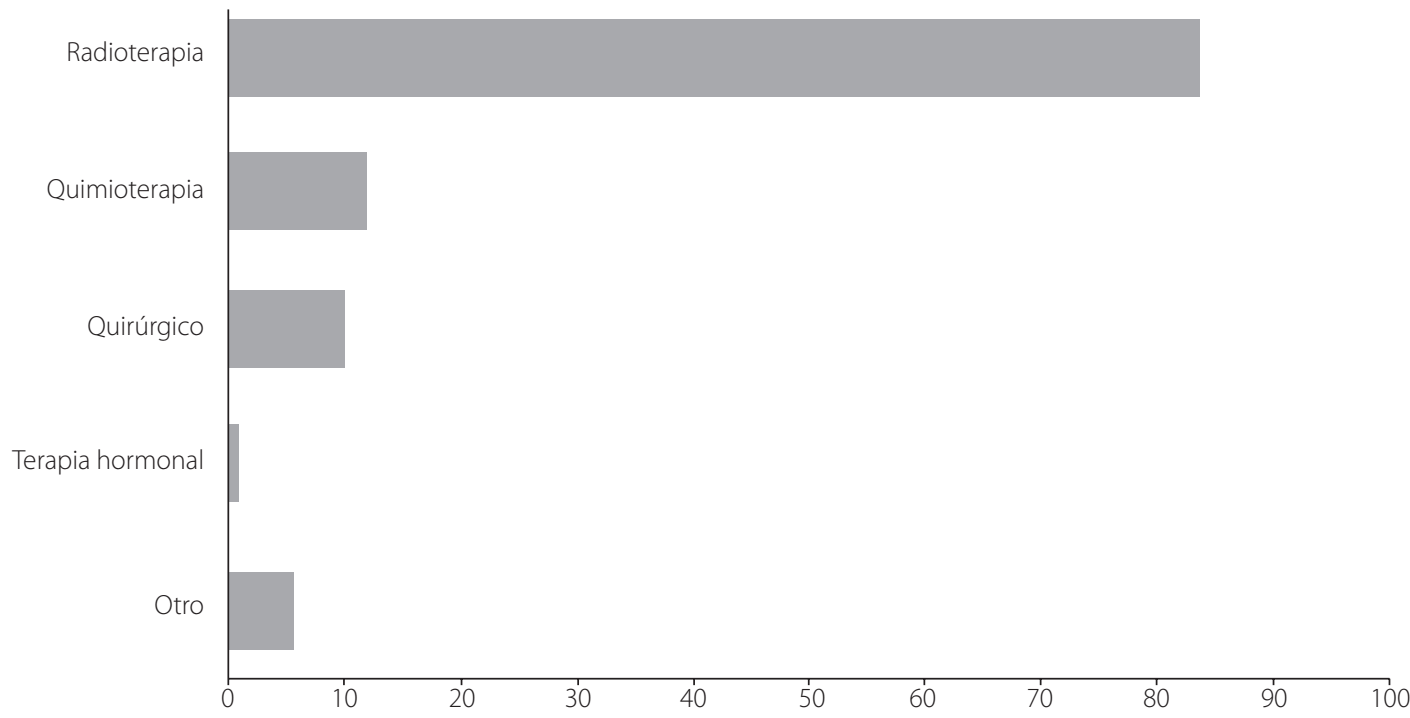

Porcentaje de tratamiento (\%)

Figura 3. Tipo de tratamiento que cumplen las pacientes con cáncer de mama ( $n=295)$. 
Según la Sociedad Americana del Cáncer, los partos disminuyen el riesgo de cáncer de mama ${ }^{9,10}$, y tener más de tres hijos está asociado con una disminución de riesgo de cáncer de mama tipo ER+22. Estudios poblacionales en Alemania sobre cáncer de mama reportaron que más de la mitad de las pacientes tenían entre uno y dos hijos ${ }^{11}$. Sin embargo, en la presente investigación difirió, con porcentajes mayores en uno y más de tres hijos; una posible explicación de esta diferencia es la cultura de los países, la tasa de fecundidad y el nivel de desarrollo: los países latinoamericanos tienen tasas de fecundidad y natalidad más altas que los países europeos ${ }^{23}$. En este estudio se observó un mayor número de hijos y a pesar de ello presencia de cáncer; este resultado debe interpretarse con cautela, ya que la edad del primer embarazo y el subtipo molecular de cáncer ${ }^{22}$ son variables relevantes para el análisis, pero ese tipo de información no se registró en su totalidad en las historias clínicas.

El signo clínico inicial más frecuente fue tumoración o masa, similar a lo reportado en estudios clínicos ${ }^{6}$ y por la Organización Mundial de la Salud (OMS)5. El tamaño del tumor en los casos incidentes en Estados Unidos desde el 2012 fue menor o igual a 2 $\mathrm{cm}^{9}$, lo que no coincide con el tamaño promedio de $4.8 \mathrm{~cm}$ encontrado en las pacientes, posiblemente por la identificación más tardía en el país, congruente con tumores más grandes.

En México, los signos como retracción de la piel y secreción de pezón fueron poco frecuentes ${ }^{8}$, similar a lo que se encontró en la presente investigación. En nuestra investigación los estadios de la enfermedad más frecuentes fueron localmente avanzados, algo que es incongruente con lo registrados en los casos incidentes de otros estudios, donde se reportó una frecuencia elevada de estadios tempranos ${ }^{6-8,12}$. Como se revisó en la variable del tamaño del tumor, puede ser explicado por una identificación de la enfermedad más tardía, y en este caso también se puede analizar el espacio de tiempo desde el diagnóstico en un centro de salud hasta la evaluación y tratamiento en el ICES.

La estadificación TNM, dividida por la categoría de T, mostró un mayor porcentaje en 2,3 y $4, \mathrm{~N}$, con mayor frecuencia en 1 y 2 ; esto difiere con lo reportado en otros países con tasa en T2 y N0, pues la captación de pacientes con cáncer sin invasión de ganglios ha aumentado conforme han transcurrido los años, posiblemente por mejoras en la identificación $n^{7,12}$. Las variables reportadas de tamaño de tumor y $\mathrm{N}$ mayor $\mathrm{O}$ igual a uno contribuyen a la clasificación de cáncer local avanzado observado en este estudio, posiblemente a causa de una identificación más retrasada, o tal vez amerite estudiar sus causas en posteriores trabajos.

La distribución en la expresión de marcadores inmunohistoquímicos en la literatura, el ER y PR se encuentran positivos en más de la mitad de los casos; el HER2 positivo es bajo, mientras que el HER2 negativo es más elevado $7,12,17,24$. En este resultado se observó un patrón de distribución de receptores moleculares similar a la evidencia; en general los cánceres de mama tuvieron una mayor frecuencia en la positividad para la expresión de receptores hormonales (ER, PR) y la negatividad para el HER2. La importancia de este resultado se relaciona con el tratamiento dirigido según el subtipo de cáncer ${ }^{25}$, así como con los factores de riesgo que se estudian en la actualidad; pero la interpretación de la distribución debe hacerse con cautela al no realizarse esta prueba en la totalidad de las pacientes.

La estirpe histológica más frecuente correspondió al carcinoma ductal invasivo, seguido en frecuencia por el carcinoma lobulillar invasivo (ILC), el carcinoma mucinoso y papilar, menos frecuente. Este distribución y la frecuencia del carcinoma ductal in situ mayor respecto al carcinoma lobular in situ es similar a lo reportado en diferentes estu$\operatorname{dios}^{7,8,24}$, lo que indica que se comparten características en el desarrollo del cáncer a nivel tisular, igual que lo reportado en el resto del mundo. En la actualidad es importante también clasificar a nivel molecular, debido a que han mostrado un comportamiento distinto según el subtipo ${ }^{26}$.

La evaluación funcional por la escala de Karnofsky de las pacientes con cáncer al momento que ingresaron al ICES fue en mayor porcentaje con la puntuación de 100 y un rango entre 80-100, lo que difiere con lo observado en estudios de calidad de vida, que registran un promedio de 88 puntos y un rango entre 40 a $100^{13}$. Una explicación puede ser que, por tratarse de un centro de atención de referencia de otros establecimientos de salud de El Salvador, las pacientes ingresaron con algún tratamiento ya iniciado.

En estudios hospitalarios, el manejo más frecuente es el quirúrgico con un tipo de cirugía por conservación de la mama ${ }^{13,14}$. En más de la mitad de los pacientes se aplica radioterapia, quimioterapia y terapia hormonal, en modalidad adyuvante ${ }^{13}$. En Estados Unidos se aplicó con mayor frecuencia cirugía más radioterapia en etapas tempranas del cáncer; en estadio III se realizó mastectomía más radioterapia y quimioterapia; en etapa IV se realizó con mayor frecuencia radioterapia y/o quimioterapia 9 . En raras ocasiones mencionan radioterapia como 
tratamiento único9. Esta evidencia no coincidió con los resultados de este estudio, ya que la terapia más frecuente fue la radioterapia tipo teleterapia como único tratamiento con respecto a los demás tipos y combinaciones de tratamientos, como quimioterapia y combinación radioterapia-cirugía, que tuvieron frecuencias bajas. Según lo reportado en otros estudios, el tumor local avanzado debe tratarse con una combinación de tratamientos de cirugía más radioterapia y quimioterapia, no solo radioterapia como único tratamiento, pero este resultado debe interpretarse en el contexto de que el ICES es un centro que brinda servicios de tratamiento enfocados en radioterapia.

En cuanto al manejo, algunos casos fueron prescritos sin tener marcadores inmunohistoquímicos, clasificación molecular o el estadio investigado por completo, un procedimiento que no es congruente con lo que se recomienda para un manejo integral y multidisciplinario del cáncer ${ }^{9,15}$.

Los expedientes clínicos consulados en este estudio registraban poca información sobre los factores de riesgo biológicos, reproductivos y estilo de vida, por lo que se dificultó ahondar en estas variables. Por otro lado, si se hubieran recabado los datos mediante entrevistas se hubiera solventado esta dificultad, pero a pesar de ello se pudo recabar información muy importante que permitió responder la pregunta de investigación.

Los resultados han dado indicios del comportamiento del cáncer de mama en El Salvador, por lo que es importante señalar que la detección temprana del cáncer de mama y el manejo oportuno son punto relevante para el control de esta enfermedad, por lo que se deben reforzar las políticas de salud con un enfoque basado en la eviden$\mathrm{cia}^{27}$, así como un tratamiento integral y adecuado ${ }^{25,26,28}$ con la potenciación de la medicina personalizada, ya que esta enfermedad puede presentar heterogeneidad según parámetros biológicos y genéticos $25,26,29$, de manera que se podría disminuir las muertes y mejorar la sobrevida de las pacientes.

Estudios adicionales que caractericen clínica, molecular y epidemiológicamente el cáncer de mama en los hospitales de El Salvador podrían revelar si el comportamiento es similar o diferente al que se ha mostrado.

\section{Conclusión}

El perfil clínico-epidemiológico de las pacientes con cáncer de mama atendidas en el ICES se ajusta a las siguientes características: adulta mayor de la sexta década de la vida, con sobrepeso, de ocupación ama de casa, con uno o más de tres hijos, procedente del área urbana, del departamento de San Salvador. Las características clínicas del cuadro inicial comprendieron tumoración con un tamaño promedio de $4 \mathrm{~cm}$, y clasificada como T mayor de 2 y N entre 1 y 2, con el diagnóstico de carcinoma ductal invasivo, en tratamiento por radioterapia. Es necesario llevar a cabo una detección oportuna de casos en estadios tempranos, con lo cual se requerirá tratamientos menos costosos y permitirá una mayor probabilidad de supervivencia. Para lograrlo es importante que los centros de atención sean dotados de los recursos para fenotipificación de los tumores, así como de las terapias adecuadas.

\section{Agradecimiento}

Al Instituto del Cáncer de El Salvador Dr. Narciso Díaz Bazán, por facilitarnos su apoyo en el desarrollo de la investigación.

\section{Financiamiento}

Fuentes propias de los autores del estudio.

\section{Referencias bibliográficas}

1. Sung H, Ferlay J, Siegel RL, Laversanne M, Soerjomataram I, Jemal A, et al. Global cancer statistics 2020: GLOBOCAN estimates of incidence and mortality worldwide for 36 cancers in 185 countries. CA Cancer J Clin. 2021;71(3): 209-249. DOI: 10.3322/ caac. 21660

2. Goldsbury DE, Yap S, Weber MF, Veerman L, Rankin N, Banks E, et al. Health services costs for cancer care in Australia: Estimates from the 45 and Up Study. PLOS ONE. 2018;13(7): e0201552. DOI: 10.1371/journal. pone.0201552

3. Ferlay J, Ervik M, Lam F, Colombet M, Mery L, Piñeros M, Znaor A, Soerjomataram I, Bray F. Global Cancer Observatory: Cancer Today. Lyon, France: International Agency for Research on Cancer. 2020. Fecha de consulta: 15 de febrero de 2021. Disponible en: https://gco.iarc.fr/today.

4. American Joint Committee on Cancer. Cancer Staging Manual (AJCC). 7ma Edición. Chicago. Springer; 2010.672 p.

5. Lakhani SR, Ellis IO, Schnitt SJ, Tan PH. WHO Classification of Tumours of the Breast. 4ta ed. Lyon. IARC Scientific Publication; 2012. $240 \mathrm{p}$.

6. Sofi NY, Jain M, Kapil U, Yadav CP. Epidemiological characteristics of breast cancer patients attending a tertiary healthcare institute in the National Capital Territory 
of India. J Cancer Res Ther. 2019;15(5):10871091. DOl: 10.4103/jcrt.JCRT_868_16

7. Ramírez-Martínez CM, Clavijo-Rodríguez JM, Estrada-Restrepo J, Restrepo-Ramírez CA. Descripción clínica, anatomopatológica y de tratamiento de pacientes con cáncer de mama en una unidad de mastología de la ciudad de Medellín, Colombia. 20062013. Rev CES Med 2015;29(2): 181-190. Disponible en: http://www.scielo.org.co/ scielo.php?script $=$ sci arttext\&pid=S0120$87052015000200003 \& \operatorname{lng}=e n$.

8. Sánchez-Ramírez B, Herrerías-Canedo T, Olaya-Guzmán EJ, Sequeira-Alvarado KA. Abordaje diagnóstico del cáncer de mama en el Instituto Nacional de Perinatología. Perinatol Reprod Hum. 2012;26(2):106-114. Disponible en: http://www.scielo.org.mx/ pdf/prh/v26n2/v26n2a5.pdf

9. American Cancer Society. Breast Cancer Facts \& Figures 2017-2018. Atlanta. American Cancer Society. 2017. 44 p. Disponible en: https://www.cancer.org/ content/dam/cancer-org/research/ cancer-facts-and-statistics/breast-cancerfacts-and-figures/breast-cancer-facts-andfigures-2017-2018.pdf

10. Lambertini M, Santoro L, Del Mastro L, Nguyen B, Livraghi L, Ugolini D, et al. Reproductive behaviors and risk of developing breast cancer according to tumor subtype: A systematic review and meta-analysis of epidemiological studies. Cancer Treat Rev. 2016;49:65-76. DOI: 10.1016/j.ctrv.2016.07.006

11. Barnes BB, Steindorf K, Hein R, Flesch-Janys D, Chang-Claude J. Population attributable risk of invasive postmenopausal breast cancer and breast cancer subtypes for modifiable and non-modifiable risk factors. Cancer Epidemiol. 2011;35(4):345-352. DOI: 10.1016/j.canep.2010.11.003

12. Schaffar R, Bouchardy C, Chappuis PO, Bodmer A, Benhamou S, Rapiti E. A population-based cohort of young women diagnosed with breast cancer in Geneva, Switzerland. PLoS One. 2019;14(9): e0222136. DOI: 10.1371/journal. pone.0222136

13. Hofsø K, Bjordal K, Diep LM, Rustøen T. The relationships between demographic and clinical characteristics and quality of life during and after radiotherapy: in women with breast cancer. Qual Life Res. 2014;23(10):2769-2777. DOl: 10.1007/ s11136-014-0736-2

14. Martínez Vázquez P, Burruchaga HJ, Abeszyc D, Forno M, De La Roza Lionel, Arias SM. Cáncer de mama localmente avanzado características y manejo en el Hospital Magdalena. 2014;33(118): 28-38. Disponible en: https://www.revistasamas.org.ar/
revistas/2014_v33_n118/Cancer\%20de\%20 mama\%20localmente.m18.pdf

15. McDonald ES, Clark AS, Tchou J, Zhang P, Freedman GM. Clinical diagnosis and management of breast cancer. J Nucl Med. 2016;57(1): 9S-16S. DOI: 10.2967/ jnumed.115.157834

16. Bray F, Colombet M, Mery L, Piñeros $M$, Znaor A, Zanetti R, et al. Cancer incidence in five continents Vol. XI. IARC Scientific Publication N. 166. Lyon, Francia. 2021. 1558 p.

17. Maffuz-Aziz A, Labastida-Almendaro S, Sherwell-Cabello S, Ruvalcaba-Limón E, Domínguez-Reyes C, JA Tenorio-Torres, et al. Supervivencia de pacientes con cáncer de mama. Análisis por factores pronóstico, clínicos y patológicos. Ginecol Obstet Mex. 2016;84(8): 498-506. Disponible en: https:// www.medigraphic.com/pdfs/ginobsmex/ gom-2016/gom168e.pdf

18. Suárez MER, Rangel YR, Pimentel LC. Caracterización de pacientes con cáncer de mama y sus familiares acompañantes. Medisur. 2018;16(1): 47-54. Disponible en: https://www.medigraphic.com/cgibin/ new/resumen.cgi?IDARTICULO=80086

19. Instituto Nacional de Salud. Encuesta nacional de enfermedades crónicas no transmisibles en población adulta de el salvador, ENECA-ELS 2015. San Salvador. Instituto Nacional de Salud; p. 252. Disponible en: http://ins.salud.gob.sv/wpcontent/uploads/2017/12/ENECA-2015.pdf

20. Barquera S, Hernández-Barrera L, Trejo B, Shamah T, Campos-Nonato I, RiveraDommarco J. Obesidad en México, prevalencia y tendencias en adultos. Ensanut 2018-19. Salud Publica Mex. 2020;62(6):682-692. DOI: 10.21149/11630

21. Endogenous Hormones and Breast Cancer Collaborative Group. Circulating sex hormones and breast cancer risk factors in postmenopausal women: reanalysis of 13 studies. Br J Cancer. 2011;105(5):709-722. DOI: 10.1038/bjc.2011.254

22. Li Cl, Beaber EF, Tang MT, Porter PL, Daling $J R$, Malone KE. Reproductive factors and risk of estrogen receptor positive, triplenegative, and HER2-neu overexpressing breast cancer among women 20-44 years of age. Breast Cancer Res Treat. 2013;137(2):579-587. DOI: 10.1007/s10549012-2365-1

23. United Nations. World population prospects 2019: highlights. Department of economic and social affairs, population division. 2019. Disponible en: https://population. un.org/wpp/Publications/Files/WPP2019 Highlights.pdf

24. Lietard C, Conan V, Le Fur J-M, Minot J-P, Le Callonec M, Brisseau C, et al. Épidémiologie des cancers du sein dans le Finistère entre 
2000 et 2009 à partir d'une base de données anatomopathologiques. Annales de Pathologie. 2013;33(1):38-48. DOI: 10.1016/j. annpat.2012.09.196

25. Waks AG, Winer EP. Breast cancer treatment: a review. JAMA. 2019;321(3): 288-300. DOI: 10.1001/jama.2018.19323

26. Marzo-Castillejo M, Vela-Vallespín C, Bellas-Beceiro B, Bartolomé-Moreno C, Melús-Palazón E, Vilarrubí-Estrella M, et al. Recomendaciones de prevención del cáncer. Actualización PAPPS 2018. Aten Primaria. 2018;50(1): 41-65. DOI: 10.1016/ S0212-6567(18)30362-7

27. Raichand S, Dunn AG, Ong M-S, Bourgeois FT, Coiera E, Mandl KD. Conclusions in systematic reviews of mammography for breast cancer screening and associations with review design and author characteristics. Syst Rev. 2017;6(1):105. DOI: 10.1186/s13643-017-0495-6

28. Lovelace DL, McDaniel LR, Golden D. Long-Term Effects of breast cancer surgery, treatment, and survivor care. J Midwifery Womens Health. 2019;64(6):713-724. DOI: 10.1111/jmwh.13012

29. Warrier S, Tapia G, Goltsman D, Beith J. An update in breast cancer screening and management. Womens Health (Lond). 2016;12(2):229-239. DOI: 10.2217/ whe. 15.105 